TITLE:

\title{
Circadian clock regulates photoperiodic responses governed by distinct output pathways in the bean bug, Riptortus pedestris
}

\section{AUTHOR(S):}

Omura, Shigeki; Numata, Hideharu; Goto, Shin G.

\section{CITATION:}

Omura, Shigeki ... [et al]. Circadian clock regulates photoperiodic responses governed by distinct output pathways in the bean bug, Riptortus pedestris. Biological Rhythm Research 2016, 47(6): 937-945

\section{ISSUE DATE:}

2016-08-02

URL:

http://hdl.handle.net/2433/218414

\section{RIGHT:}

This is an Accepted Manuscript of an article published by Taylor \& Francis in 'Biological Rhythm Research' on 02 August 2017, available online: http://www.tandfonline.com/10.1080/09291016.2016.1212515.; The full-text file will be made open to the public on 02 Aug 2017 in accordance with publisher's 'Terms and Conditions for Self-Archiving'.; This is not the published version. Please cite only the published version.; この論文は出版社版でありません。引用の際には出版社 版をご確認ご利用ください。 
Circadian clock regulates photoperiodic responses governed by distinct output pathways in the bean bug, Riptortus pedestris

Shigeki Omuraa, Hideharu Numata ${ }^{\mathrm{b}}$ and Shin G. Goto ${ }^{\mathrm{a}}$

${ }^{a}$ Graduate School of Science, Osaka City University, Osaka, Japan; ${ }^{b}$ Graduate School of Science, Kyoto University, Kyoto, Japan

* Corresponding author. E-mail: shingoto@sci.osaka-cu.ac.jp

Shigeki Omura and Shin G. Goto: Graduate School of Science, Osaka City University, Sumiyoshi-ku, Osaka 558-8585, Japan. Tel. +81-6-6605-2573, Fax $+81-6-6605-2522$.

Hideharu Numata: Graduate School of Science, Kyoto University, Sakyo-ku, Kyoto 606-8502, Japan. Tel. +81-75-753-4073, Fax +81-75-753-4113.

E-mail addresses: 0mura0210@gmail.com (S. Omura), numata@ethol.zool.kyoto-u.ac.jp (H. Numata), shingoto@sci.osaka-cu.ac.jp (S.G. Goto).

Acknowledgments

We thank Dr. Y. Suzaki (Okayama University, Japan), who kindly provided us with the R. pedestris colony. This study was supported by JSPS KAKENHI (Grant-in-Aid for Scientific Research B) Grant Number 26292175 to H.N. 


\section{Circadian clock regulates photoperiodic responses governed by distinct} output pathways in the bean bug, Riptortus pedestris

Effects of RNA interference (RNAi) targeted against circadian clock genes on two distinct types of photoperiodic responses-ovarian development and lipid accumulation - were investigated in a bean bug $R$. pedestris, to explore which physiological process in the photoperiodic response involved the circadian clock. Ovarian development and lipid accumulation are known to be regulated by distinct output pathways. Control insects showed clear photoperiodic responses; i.e., induction of ovarian development and suppression of lipid accumulation under long-day conditions, whereas opposite characteristics under short-day conditions. We found that RNAi directed against period, a negative element of the circadian clock, produced a long-day effect for both of ovarian development and lipid accumulation, while RNAi directed against Clock, a positive element of the circadian clock, produced a short-day effect for both, irrespective of photoperiod. These results indicate that the circadian clock comprised of these genes regulates a process governing both distinct photoperiodic responses.

Keywords: Circadian clock · diapause $\cdot$ lipid content · ovarian development · photoperiodism 


\section{Introduction}

Most organisms inhabiting the temperate zone have evolved photoperiodism, an adaptive, seasonal timing system that enables them to coordinate their developmental and physiological processes to seasonal changes in the environment in response to day length (photoperiod) as a cue (Saunders 2002). Many insect species also show photoperiodism and enter diapause, hormonally-regulated developmental or reproductive arrest with additional energy reserves, in response to autumnal short days (Danks 1987; Tauber et al. 1986). Bünning (1936) first proposed the functional involvement of the circadian clock in photoperiodic time measurement, which assesses day or night length (Saunders 2002). His idea was further developed by several researchers. As a result, the basic concept of a circadian clock in photoperiodic time measurement, the Bünning hypothesis, is widely accepted for insects (Saunders and Bertossa 2011) and other organisms (Nelson et al. 2010). All mechanisms regulating photoperiodism are assumed to be in the brain in insects (Bowen et al. 1984; Hasegawa and Shimizu 1987); therefore, the circadian clock involved in the photoperiodic time measurement system is expected to also reside in the brain.

Although the molecular mechanisms of the circadian clock underlying insect photoperiodism have long been unknown, those of the circadian system governing locomotor activity rhythms are well studied in several species (reviewed by Tatarogu and Emery 2015; Tomioka and Matsumoto 2015). The insect circadian clock is composed of a number of circadian clock genes, including period (per), timeless (tim), cycle (cyc), Clock (Clk), mammalian-type cryptochrome (cry-m, also known as cry2), and Par-domain protein 1 (Pdp1), in interlocked negative feedback loops. In the loops, protein products of $c y c$ and $C l k$, positive elements, activate the transcription of per, tim, and many other clock-controlled genes, whereas protein products of per, tim, and cry-m, negative 
elements, suppress the CYC-CLC activity.

In some insect species, gene knock-down by RNA interference (RNAi) reveals causal involvement of these clock genes in photoperiodism (reviewed by Numata et al. 2015). For example, RNAi directed against per, cry-m, cyc, and Clk disrupts the photoperiodic regulation of ovarian development in females of Riptortus pedestris (F.), the focal species of this study (Ikeno et al. 2010, 2011, 2013). RNAi directed against per, tim, and $c r y$-m, and pigment-dispersing factor $(p d f)$, a putative output gene of the circadian clock, also disrupts the photoperiodic response in the Northern house mosquito Culex pipiens L. (Meuti et al. 2015). RNAi directed against per disrupts photoperiodic induction of larval diapause in the jewel wasp Nasonia vitripennis (Walker) (Mukai and Goto 2016). In addition, per RNAi disrupts photoperiodic regulation of nymphal development as well as circadian locomotor rhythmicity in the cricket Modicogryllus siamensis Chopard (Sakamoto et al. 2009). It is still possible that the effects of RNAi targeted against clock genes are not mediated by the malfunction of the circadian clock as a functional unit, but are mediated by the malfunction of individual genes, i.e., gene pleiotropy (Emerson et al. 2009). However, a series of experiments in $R$. pedestris and Drosophila melanogaster Meigen supports the involvement of the clock as a module, and not as individual genes, in photoperiodism (Goto 2013; Pegoraro et al. 2014).

Based on the published data, an important question arises: which physiological process in photoperiodic responses is affected by malfunction of the circadian clock? According to the Bünning hypothesis, the circadian clock is involved in the photoperiodic time measurement system. Alternatively, malfunction of peripheral clocks, which reside in a variety of organs such as the compound eyes, antennae, wings, legs, Malpighian tubules, and epidermis (Tomioka et al. 2012; Ito and Tomioka 2016) may affect output processes in the 
photoperiodic cascade, including endocrine effector regulation. The significance of cross-talk between the brain and a peripheral tissue in diapause is proposed in the moth Helicoverpa armigera (Hübner) (Xu et al. 2012). In experiments where RNAi is introduced into an organism by feeding or injection, gene knock-down is not tissue- or cell type-specific but is systemic. Therefore, it is not easy to know if malfunction of the central clock or malfunction of a peripheral clock results in an abnormality in photoperiodism (Bradshaw and Holzapfel 2010).

Here, we approached this issue in $R$. pedestris with special interest in photoperiodic responses under the control of distinct endocrine effectors, i.e., ovarian development and lipid accumulation. This species shows clear photoperiodic responses; adult females develop their ovaries and accumulate a smaller amount of lipids under long-day conditions, whereas they suppress ovarian development and accumulate a larger amount of lipids under short-day conditions (Numata and Hidaka 1982; Morita et al. 1999). In general, photoperiodic regulation of reproductive arrest is primarily caused by reduced activity of the corpus allatum (CA), which secretes juvenile hormone (JH) (Denlinger et al. 2012). Developmental suppression of the ovary in R. pedestris is due to inactivity of the CA (Numata and Hidaka 1984; Morita and Numata 1997). In contrast, photoperiodic regulation of lipid accumulation is independent of the CA (Morita et al. 1999). Thus, reproductive organ development and lipid accumulation are regulated by distinct endocrine effectors in this species.

In the present study, we performed RNAi directed against per and Clk, the negative and positive elements in the circadian clock, respectively. In $R$. pedestris, per and Clk RNAi arrest the circadian clock at distinct phases and produce distinct output signals (Ikeno et al. 2010, 2013). RNAi targeted against per and Clk also disrupt photoperiodic induction of ovarian development, but 
their phenotypes are different (Ikeno et al. 2010, 2013): per knock-down causes a "long-day effect" that promotes ovarian development, whereas Clk knock-down causes a "short-day effect" that suppresses ovarian development, irrespective of photoperiod. If per and Clk RNAi show the long-day and short-day effects, respectively, in both ovarian development and in lipid accumulation, then the clock would be involved in a central process in photoperiodism, which governs both events.

\section{Materials and methods}

\section{Insects}

A colony of $R$. pedestris, derived from individuals collected in Fukuyama, Japan $\left(34.5^{\circ} \mathrm{N}, 133.4^{\circ} \mathrm{E}\right)$ in 2006 , was kindly provided by Dr. Y. Suzaki (Okayama University, Japan). Insects were reared under short-day (LD 12:12 h) or long-day (LD 16:8 h) conditions at $25.0 \pm 1.0^{\circ} \mathrm{C}$, and fed soybean grain and water containing $0.05 \%$ sodium ascorbate and $0.025 \%$ L-cysteine (Kamano 1991).

\section{Reproductive status}

The abdomens of insects were dissected in saline $(0.9 \% \mathrm{NaCl})$ under a stereoscopic microscope at the age of 20 days after adult emergence. Insects were classified as reproductive or non-reproductive (diapause) based on ovarian development, as described previously (Numata and Hidaka 1982): females with a light-blue yolk deposition in the oocytes were judged to be reproductive and those with no deposition were judged to be non-reproductive.

\section{Lipid content}


Lipid content was estimated at the age of 20 days after adult emergence according to Morita et al. (1999) with some modifications. In brief, the body was dried for $24 \mathrm{~h}$ at $100^{\circ} \mathrm{C}$ after removal of the legs, wings, the antennae, and ovaries. Initial dry weight was measured on an electronic balance, GR-60 (A\&D Company, Tokyo, Japan). Thereafter, lipids were extracted with a chloroform and methanol (2:1) solution for $24 \mathrm{~h}$ at room temperature. After further extraction with new chloroform and methanol solution for $6 \mathrm{~h}$ at room temperature, dry weight was measured again. The difference between the initial dry weight and the dry weight after lipid extraction was regarded as the weight of the stored lipids. Lipid content was designated as the percentage of the weight of stored lipids to the initial dry weight. This simple gravimetric determination is recognized as a suitable method for routine analyses (Phillips et al. 1997).

\section{RNAi}

RNA was extracted from the whole body of $R$. pedestris with Trizol Reagent (Life Technologies, Foster City, CA, USA) and purified using Purelink Micro kit (Life Technologies). cDNA was synthesized using High Capacity cDNA Reverse Transcription kit (Life Technologies). T7-promoter-attached DNA fragments of per and Clk were amplified by PCR with the primers shown in Table 1 and Pwo Super Yield Polymerase (Roche, Basel, Switzerland). Double-stranded (ds) RNA was synthesized by T7 Ribomax Express RNAi System (Promega, Fitchburg, WI, USA) according to the supplier's instructions. As a control, dsRNA from $\beta$-lactamase (bla), which provides bacteria with ampicillin resistance, was also synthesized using pGEM-T Easy Vector (Promega).

One microgram of each dsRNA in $1 \mu \mathrm{l}$ of saline was injected into the head of adult insects reared under short-day conditions within $24 \mathrm{~h}$ after adult 
emergence. Thereafter, the insects were continuously maintained under short-day conditions or transferred to long-day conditions. It is important to note that the protocol in the present study is the same as that described in Ikeno et al. (2010, 2013). These studies verified that injection of per and Clk dsRNA effectively suppresses per and Clk expression, respectively, in R. pedestris.

\section{Results}

\section{Effects of RNAi targeted against circadian clock genes on reproductive status}

Intact females showed a clear photoperiodic response in the reproductive status (Figure 1(a) and 1(c)), i.e., approximately $90 \%$ of intact females were non-reproductive under short-day conditions (i.e., diapause), whereas all females became reproductive after they were transferred to long-day conditions. The same holds true in the control (bla) dsRNA-injected individuals. However, all females injected with per dsRNA were reproductive and most females injected with Clk dsRNA were non-reproductive (diapause), irrespective of photoperiod.

\section{Effects of RNAi targeted against circadian clock genes on lipid content}

After observing the reproductive status of these individuals, their lipid contents were measured (Figure 1(b) and 1(c)). A clear photoperiodic response was observed in intact females; lipid content of females was approximately 55\% under short-day conditions and 45\% under long-day conditions. The same holds true for dsbla RNA-injected individuals. By contrast, dsper RNA-injected insects accumulated less lipid not only under long-day conditions, but also under short-day conditions; the level was comparable with that of intact and dsbla RNA-injected control insects under long-day conditions. In dsClk 
RNA-injected insects, lipid accumulation was higher, irrespective of photoperiod, and the result was comparable to that in intact and control dsRNA-injected individuals under short-day conditions.

\section{Discussion}

It is well-known that most insects in diapause store additional energy reserves. Triacylglycerides are the dominant form of energy storage. They often account for as much as $80-95 \%$ of total lipid content, because of their high caloric content, low hydration state and possible high yield of metabolic water (Hahn and Denlinger 2011). The fat body is the primary site of triacylglyceride production and the storage site in insects. We removed ovaries before the quantification of lipids in the present study; therefore, the extracted lipids were mostly from the fat body.

The present study revealed that $R$. pedestris has clear photoperiodic responses in ovarian development and lipid accumulation, as reported in the previous studies (Numata and Hidaka 1982; Morita et al. 1999). Numata and Hidaka (1984) demonstrated that topical application of a JH analogue stimulates ovarian development in females in diapause. Allatectomy (surgical removal of the CA) suppresses ovarian development even under long-day conditions, and transection of the nervi corporis allati (NCA), the neural pathway from the brain to the $\mathrm{CA}$, induces ovarian development under short-day conditions (Morita and Numata 1997). These results indicate that suppression of ovarian development is due to the brain's inhibition of the CA via the NCA, thereby preventing the CA from secreting JH. By contrast, allatectomy and transection of the NCA do not disrupt photoperiodic regulation of lipid accumulation in R. pedestris (Morita et al. 1999). Thus, 
although the endocrine factor is yet unidentified, it is evident that the photoperiodic regulation of lipid accumulation is independent of $\mathrm{JH}$ and CA.

In the present study, RNAi directed against the negative element (per) and the positive element $(\mathrm{Clk})$ induces phenotypes comparable to control insects under long-day conditions and short-day conditions, respectively, not only for ovarian development but also for lipid accumulation. Thus, per RNAi and Clk RNAi show long- and short-day effects, respectively, for these two photoperiodic responses, which are under the control of distinct endocrine effectors (Morita and Numata 1997; Morita et al. 1999). Pegoraro and co-authors focused on the photoperiodic response in chill coma recovering time (CCRt) in D. melanogaster and found that mutants with long free-running periods consistently show short-day type responses in CCRt under both long and short photoperiods, compared with mutants with short free-running periods (Pegoraro et al. 2014). Ikeno and co-authors found in the photoperiodic induction of ovarian development in $R$. pedestris that the circadian clock is involved in an upstream cascade of $\mathrm{JH}$ secretion, but neither in ovarian development itself nor in a downstream cascade of JH secretion (Ikeno et al. 2010). We do not exclude a possibility of involvement of the peripheral clock in regulation of lipid accumulation itself; nevertheless, the present study, together with the previous studies, suggests that the circadian clock plays a pivotal role in a central process in the photoperiodic cascade, which regulates both photoperiodic responses in R. pedestris.

Recently, Urbanová and co-authors focused on the role of the clock genes in photoperiodic regulation of the male reproductive organ (accessary gland), locomotor activity, and mating behaviour in P. apterus and reached similar conclusions (Urbanová et al. 2016). In this species, long-day conditions induce development of the accessary gland and higher locomotor activity, 
whereas short-day conditions suppress them. When males under short-day conditions were transferred to long-day conditions, their reproductive organs started to develop. Although Clk RNAi did not affect reproduction of males reared continuously under long-day conditions, it destroyed the ability of short-day males to switch to the reproductive mode after the transfer to a long-day regime. RNAi targeted against $c r y$ - $m$ also disrupted discrimination of long days from short days. These results indicate that the circadian clock functions in photoperiodic time measurement. Interestingly, high locomotor activity of males reared under long-day conditions and low activity of males under short-day conditions are independent of JH (Urbanová et al. 2016). Activity of short-day males gradually increased when they were transferred to long-day conditions, but the locomotor activity of Clk RNAi males remained low after the transfer. Thus, Clk RNAi disrupted not only JH-dependent photoperiodic regulation of the reproductive organ, but also JH-independent photoperiodic regulation of locomotor activity level (Urbanová et al. 2016). These results, together with the results in D. melanogaster (Pegoraro et al., 2014) and the present results in $R$. pedestris, support the role of the circadian clock in photoperiodic time measurement. However, we still do not know exactly how the circadian clock is involved in the photoperiodic time measurement. Although more than a dozen hypothetical photoperiodic time measurement models have been proposed by incorporating accumulated experimental data under natural and unnatural photoperiodic conditions (Vaz Nunes and Saunders 1999; Saunders 2002), molecular mechanisms underlying them are still largely veiled. Clarifying the mechanisms is the next step to be addressed.

\section{Acknowledgments}


We thank Dr. Y. Suzaki (Okayama University, Japan), who kindly provided us with the $R$. pedestris colony. This study was supported by JSPS KAKENHI (Grant-in-Aid for Scientific Research B) Grant Number 26292175 to H.N.

\section{Disclosure statement}

No potential conflict of interest was reported by the authors.

\section{References}

Barthel A, Schmoll D, Unterman TG (2005) FoxO proteins in insulin action and metabolism. Trends Endocrinol Metab 16:183-189. doi:10.1016/j.tem.2005.03.010

Bowen MF, Saunders DS, Bollenbacher WE, Gilbert LI (1984) In vitro reprogramming of the photoperiodic clock in an insect brain-retrocerebral complex. Proc Natl Acad Sci USA 81:5881-5884. doi:10.1073/pnas.81.18.5881

Bradshaw WE, Holzapfel CM (2010) Circadian clock genes, ovarian development and diapause. BMC Biol 8:115. doi: 10.1186/1741-7007-8-115

Bünning E (1936) Die Endonome Tagesrhythmik als Grundlage der Photoperiodischen Reaktion. Ber Dtsch Bot Ges 54:590-607.

Danks HV (1987) Insect Dormancy: An Ecological Perspective. Biological Survey of Canada, Ottawa.

Denlinger DL, Yocum GD, Rinehart JP (2012) Hormonal control of diapause. In: Gilbert LI (ed) Insect Endocrinology. Academic Press, London, pp 430-463. doi: 10.1016/B978-0-12-384749-2.10010-X

Emerson KJ, Bradshaw WE, Holzapfel CM (2009) Complications of complexity: integrating environmental, genetic and hormonal control of insect diapause. 
Trends Genet 25:217-225. doi: 10.1016/j.tig.2009.03.009

Goto SG (2013) Roles of circadian clock genes in insect photoperiodism.

Entomol Sci 16:1-16. doi: 10.1111/ens.12000

Hahn DA, Denlinger DL (2010) Energetics of insect diapause. Annu Rev

Entomol 56:103-121. doi: 10.1146/annurev-ento-112408-085436

Hasegawa K, Shimizu I (1987) In vivo and in vitro photoperiodic induction of

diapause using isolated brain-suboesophageal ganglion complexes of the silkworm, Bombyx mori. J Insect Physiol 33:959-966.

doi:10.1016/0022-1910(87)90008-4

Ikeno T, Tanaka SI, Numata H, Goto SG (2010) Photoperiodic diapause under the control of circadian clock genes in an insect. BMC Biol 8:116. doi: $10.1186 / 1741-7007-8-116$

Ikeno T, Numata H, Goto SG (2011) Photoperiodic response requires mammalian-type cryptochrome in the bean bug Riptortus pedestris. Biochem Biophys Res Commun 410:394-397. doi: 10.1016/j.bbrc.2011.05.142

Ikeno T, Ishikawa K, Numata H, Goto SG (2013) Circadian clock gene Clock is involved in the photoperiodic response of the bean bug Riptortus pedestris. Physiol Entomol 38:157-162. doi: 10.1111/phen.12013

Ito C, Tomioka K (2016) Heterogeneity of the peripheral circadian systems in Drosophila melanogaster: A review. Front Physiol 7:8. doi: 10.3389/fphys.2016.00008

Kamano S (1991) Riptortus clavatus (Thunberg) (Bean bug). In: Yushima T, Kamano S, Tamaki Y (Eds) Rearing Methods of Insects. Japan Plant Protection Association, Tokyo, (in Japanese), pp 46-49

Lee RYN, Hench J, Ruvkun G (2001) Regulation of C. elegans DAF-16 and its human ortholog FKHRL1 by the daf-2 insulin-like signaling pathway. Curr Biol 11:1950-1957. doi:10.1016/S0960-9822(01)00595-4 
Meuti ME, Stone M, Ikeno T, Denlinger DL (2015) Functional circadian clock genes are essential for the overwintering diapause of the Northern house mosquito, Culex pipiens. J Exp Biol 218:412-422. doi: 10.1242/jeb.113233

Morita A, Numata H (1997) Role of the neuroendocrine complex in the control of adult diapause in the bean bug, Riptortus clavatus. Arch Insect Biochem Physiol 35:347-355. doi: 10.1002/(SICI)1520-6327(199705)35:3<347::AID-ARCH8>3.0.CO;2-Q

Morita A, Soga K, Hoson T, Kamisaka S, Numata H (1999) Changes in mechanical properties of the cuticle and lipid accumulation in relation to adult diapause in the bean bug, Riptortus clavatus. J Insect Physiol 45:241-247. doi: 10.1016/S0022-1910(98)00119-X

Mukai A, Goto SG (2016) The clock gene period is essential for the photoperiodic response in the jewel wasp Nasonia vitripennis (Hymenoptera:

Pteromalidae). Appl Entomol Zool 51:185-194. doi:

10.1007/s13355-015-0384-1

Nelson RJ, Denlinger DL, Somers DE (2009) Photoperiodism: the Biological calendar. Oxford University Press, Oxford.

Numata H, Hidaka T (1982) Photoperiodic control of adult diapause in the bean bug, Riptortus clavatus Thunberg (Heteroptera: Coreidae). I. Reversible induction and termination of diapause. Appl Entomol Zool 17:530-538

Numata H, Hidaka T (1984) Termination of adult diapause by a juvenile hormone analogue in the bean bug, Riptortus clavatus. Zool Sci 1, 751-754.

Numata H, Miyazaki Y, Ikeno T (2015) Common features in diverse insect clocks. Zool Lett 1:10. doi: 10.1186/s40851-014-0003-y

Pegoraro M, Gesto JS, Kyriacou CP, Tauber E, 2014 Role for circadian clock genes in seasonal timing: testing the Bünning hypothesis. PLoS Genet 10:e1004603. doi: 10.1371/journal.pgen.1004603 
Phillips KM, Tarragó-Trani MT, Grove TM, Grün I, Lugogo R, Harris RF, Stewart KK (1997) Simplified gravimetric determination of total fat in food composites after chloroform-methanol extraction. J Am Oil Chemists' Soc 74:137-142. doi: 10.1007/s11746-997-0158-1

Sakamoto T, Uryu O, Tomioka K (2009) The clock gene period plays an essential role in photoperiodic control of nymphal development in the cricket Modicogryllus siamensis. J Biol Rhythms 24:379-390. doi: $10.1177 / 0748730409341523$

Saunders DS (2002) Insect Clocks, third ed. Elsevier, Amsterdam.

Saunders DS, Bertossa RC (2011) Deciphering time measurement: the role of circadian 'clock' genes and formal experimentation in insect photoperiodism. J Insect Physiol 57:557-566. doi: 10.1016/j.jinsphys.2011.01.013

Tataroglu O, Emery P (2015) The molecular ticks of the Drosophila circadian clock. Curr Opin Insect Sci 7:51-57. doi: 10.1016/j.cois.2015.01.002

Tauber MJ, Tauber CA, Masaki S (1986) Seasonal adaptations of insects. Oxford University Press, Oxford.

Tomioka K, Matsumoto A (2015) Circadian molecular clockworks in non-model insects. Curr Opin Insect Sci 7:58-64. doi: 10.1016/j.cois.2014.12.006

Tomioka K, Uryu O, Kamae Y, Umezaki Y, Yoshii T (2012) Peripheral circadian rhythms and their regulatory mechanism in insects and some other arthropods: a review. J Comp Physiol B 182:729-740. doi: $10.1007 / \mathrm{s} 00360-012-0651-1$

Urbanová V, Bazalová O, Vaněčková H, Dolezel D (2015) Photoperiod regulates growth of male accessory glands through juvenile hormone signaling in the linden bug, Pyrrhocoris apterus. Insect Biochem Mol Biol 70:184-190. doi: 10.1016/j.ibmb.2016.01.003. 
Vaz Nunes M, Saunders D (1999) Photoperiodic time measurement in insects: a review of clock models. J Biol Rhythms 14:84-104. doi:

$10.1177 / 074873049901400202$

Xu W-H, Lu Y-X, Denlinger DL (2012) Cross-talk between the fat body and brain regulates insect developmental arrest. Proc Natl Acad Sci USA 109:14687-14692. doi: 10.1073/pnas.1212879109

Zar JH (2010) Biostatistical analysis, 5th edn. Prentice-Hall, Upper Saddle River 
Table 1 Sequence of primers

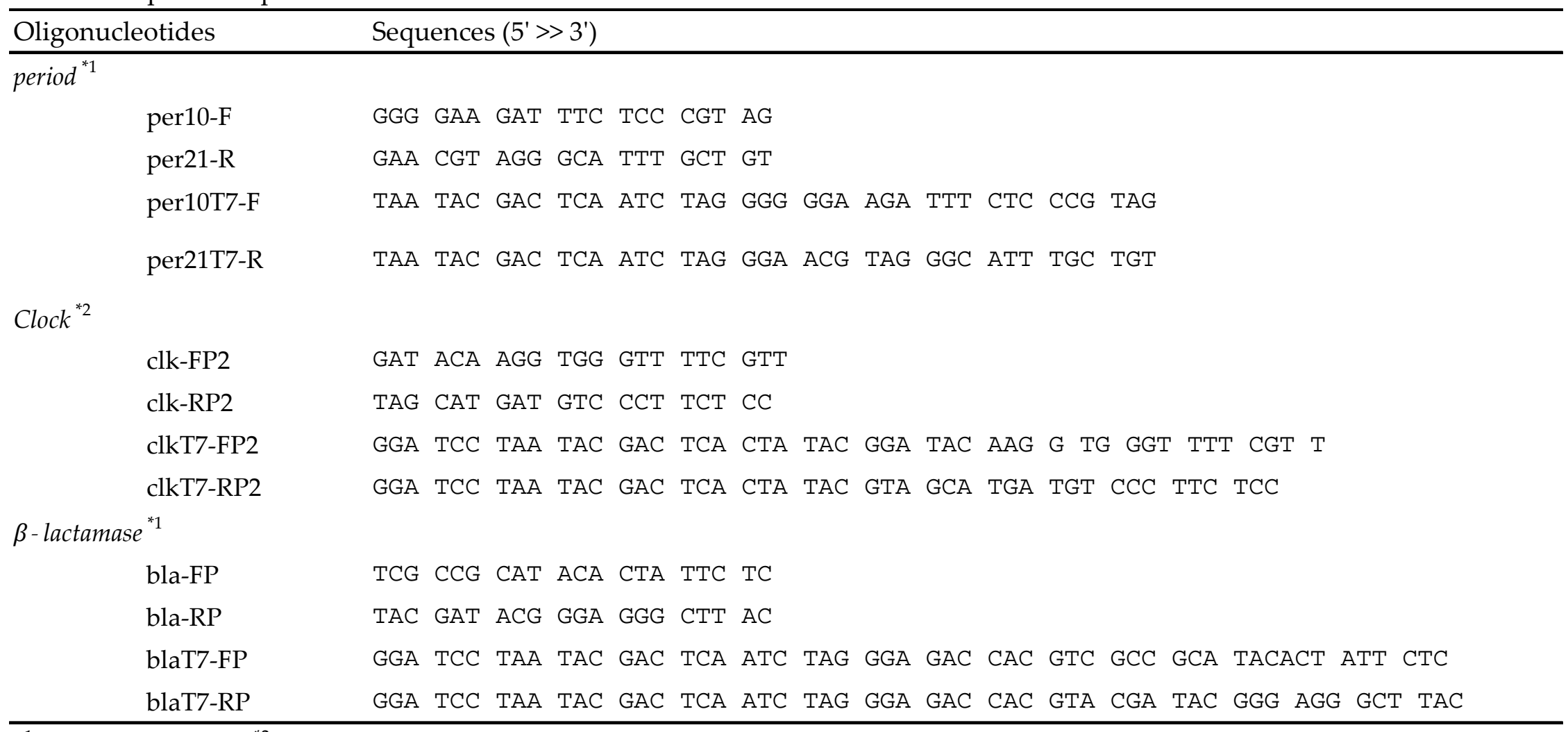

${ }^{* 1}$ Ikeno et al. (2010), ${ }^{* 2}$ Ikeno et al. (2013) 
(a)

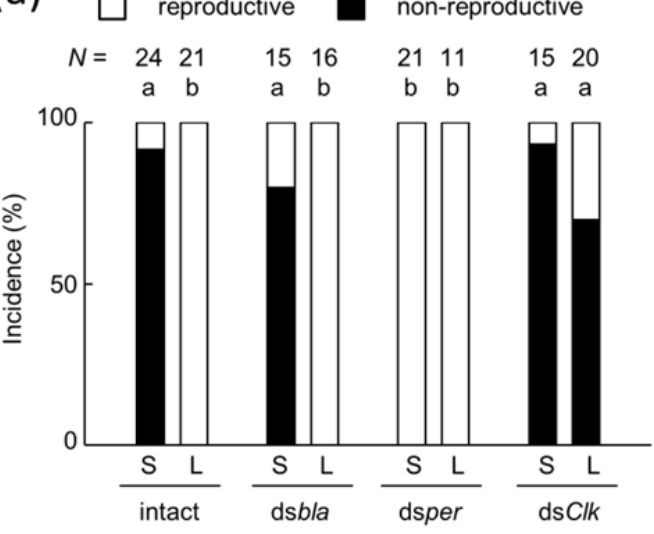

(c)

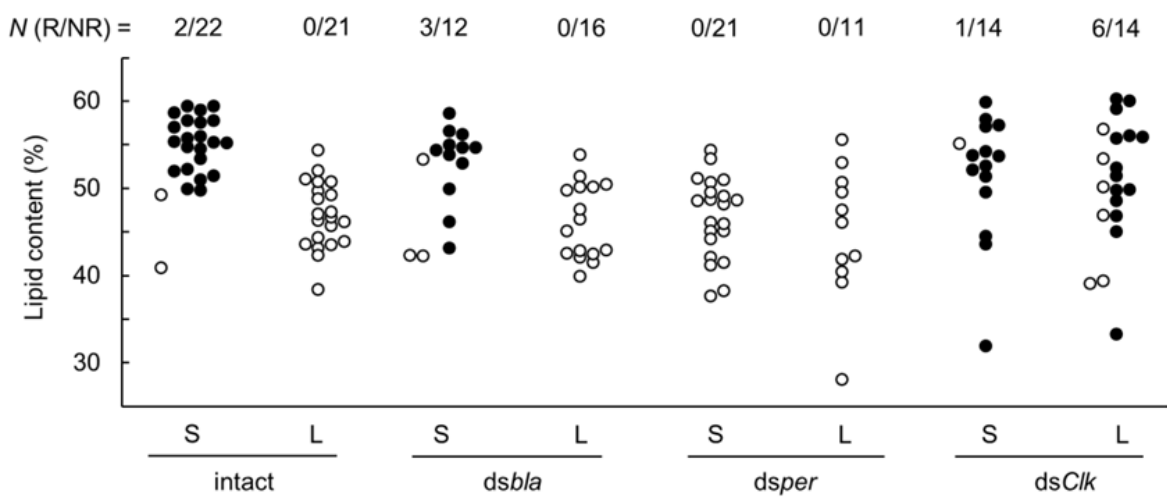

(b)

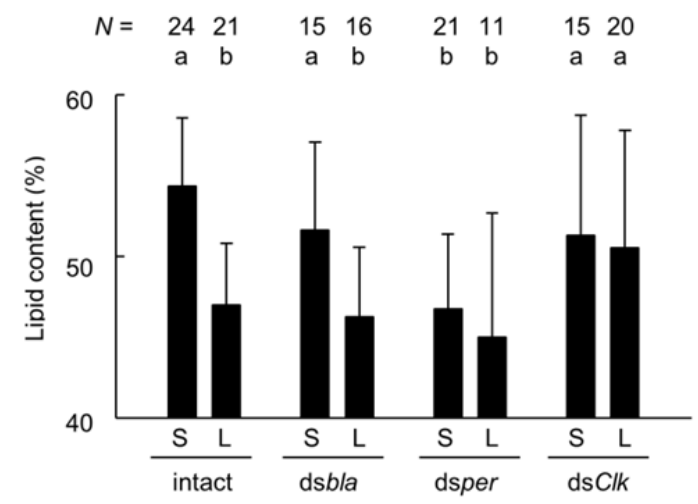


under short-day (S) or long-day (L) conditions. (a) Ovarian development. Open and closed columns indicate reproductive and non-reproductive females, respectively. Different letters indicate significant differences (Tukey-type multiple comparison test for proportions, $P<0.05$; Zar 2010). (b) Lipid content (mean \pm S.D.). Different letters indicate significant differences (Steel-Dwass test, $P<0.05$; Zar 2010). (c) Lipid content and ovarian development of each individual. Data are from $a$ and $b$. Open circles and " $\mathrm{R}$ " on the top indicate reproductive individuals, and closed circles and "NR" indicate non-reproductive individuals. 